\title{
A Survey of Bluetongue Infection and Associated Risk Factors among the One-Humped Camel (Camelus dromedaries) in Gadarif State, Eastern Sudan
}

\author{
Hatim H. Abraheem $\left(\mathbb{D},{ }^{1}\right.$ Amira M. Elhassan $\left(\mathbb{D},{ }^{1}\right.$ Mohammed O. Hussien $\left(\mathbb{D},{ }^{2}\right.$ \\ Khalid A. Enan $\mathbb{D}^{,},{ }^{2}$ Azza B. Musa $\mathbb{1}^{2},{ }^{2}$ and Abdel Rahim M. El Hussein $\mathbb{( \mathbb { D }}^{1}$ \\ ${ }^{1}$ Central Veterinary Research Laboratory (CVRL), Animal Resources Research Corporation (ARRC), P.O. Box 8067, El Amarat, \\ Khartoum, Sudan \\ ${ }^{2}$ Central Laboratory, Ministry of Higher Education and Scientific Research, P.O. Box 7099, Khartoum, Sudan
}

Correspondence should be addressed to Hatim H. Abraheem; hatimhmad10@yahoo.com

Received 5 November 2020; Revised 22 February 2021; Accepted 17 March 2021; Published 24 March 2021

Academic Editor: Bockline Omedo Bebe

Copyright $\odot 2021$ Hatim H. Abraheem et al. This is an open access article distributed under the Creative Commons Attribution License, which permits unrestricted use, distribution, and reproduction in any medium, provided the original work is properly cited.

\begin{abstract}
Bluetongue (BT) is an infectious, noncontagious, vector-borne viral disease that affects wild and domestic ruminants transmitted by Culicoides spp. A cross-sectional study was carried out during the period 2016-2017 in Gadarif state. A total of 276 sera samples were collected from camels in six localities of Gadarif state, eastern Sudan, to investigate bluetongue virus (BTV) seroprevalence and associated risk factors of BTV infection including age, sex, breed, locality, and ecology of the region. Enzyme-linked immunosorbent assay (ELISA) was used for estimation of BTV seroprevalence rate. The overall BTV seroprevalence rate was $96.7 \%$ in the study area ranging from $93.5 \%$ to $100 \%$ in six screened localities with no significant differences. The findings revealed similar BTV seroprevalence rates in both males and females, but high rates were found in age group of less than one year and two to three years with estimated $100 \%$. However, the lowest seroprevalence was found in the age group of five to four years with estimated BTV to be $92.3 \%$. BTV seropositivity was not found to be statistically associated with examined different camel breeds which revealed 93\%, 94.4\%, 97.6\%, and 97.8\% seroprevalence in Bushari, Rashide, Arabi, and Anafi, breeds, respectively. Epidemiology of BTV assessment according to the ecology of the area showed high BTV seroprevalence in desert and savanna with estimated $100 \%$ and lower BTV seroprevalence in arid and rich savanna with estimated $94.8 \%$ and $95.7 \%$, respectively. There was no significant association between BTV ELISA positivity and sex, breed, and ecology of the area.
\end{abstract}

\section{Introduction}

The total camel population in the world is 19 million, of which, 17 million are dromedaries and 2 million are Bactrian [1]. The population of camels in Sudan is 3.3 million $[2,3]$ of which 564,756 heads are in Gadarif state. Many tribes in different parts of the Sudan depend entirely on camels for their livelihood $[4,5]$. Several studies in various countries have reported on the widespread infection of camels with bluetongue virus. These infections are mostly unapparent infection with no noticeable signs or symptoms. Bluetongue virus (BTV) is a double-stranded RNA virus (family
Reoviridae, genus Orbivirus) that causes bluetongue in ruminants with at least 28 recognized serotypes. Clinical signs of the disease include fever, nasal discharge, excessive salivation, facial edema, ulceration, cyanosis of tongue (bluetongue), coronitis, and skeletal muscle damage. Severe clinical signs are observed in sheep, and mild symptoms are usually shown in cattle, goat, camelids, and carnivores [6-8]. Culicoides midges are the main vectors of the virus, with C. imicola being the main vector species in Africa and southern Europe.

Earlier, Abu [9] conducted a serological survey in the one-humped camel in Sudan using agar gel immune- 
diffusion (AGID) test which revealed 40\%, 19.2\%, 8.7\%, $4.3 \%, 6 \%$, and $0 \%$ positivity in Al Fashir, Nyala, Tampol, Kassala, Gadarif, and Sennar, respectively, with an overall seroprevalence of $16.6 \%$. On the other hand, seroprevalence surveys of BTV in camel conducted in Khartoum and Kassala states revealed $66.8 \%$ and $12.7 \%$ seroprevalence rates, respectively $[10,11]$. However, other serological surveys showed that the antibodies against BTV were widespread in livestock species such as goats, cattle, and sheep in the country $[9,11,12]$.

The aim of this investigation was to determine the prevalence of BTV antibodies among different camel breeds and assess the risk factors predisposing the animals to BTV infection in Gadarif state, eastern Sudan.

\section{Materials and Methods}

2.1. Study Area. This study was carried out in Gadarif state which lies between $16.14^{\circ}$ altitude and $33.35^{\circ}$ longitude (Figure 1). The climate is hot in the summer. The rainy season extends four months, with an average of annual rainfall of 700 to $900 \mathrm{~mm}$. Average temperature ranges between 21.2 and $36.5^{\circ} \mathrm{C}$, whereas average relative humidity is around $43 \%$. Gadarif state is boarded by five states and one neighboring country. The study area is divided into six localities.

2.2. Study Design. This is a cross-sectional study that was carried out during the period 2016-2017. A total of 276 samples were randomly collected from camels in six localities including three localities from north section (Gadarif, central Gadarif, and Butana) and one from east (Fashaga). The method was random selection of villages from each of the 6 localities mentioned above, and the strategy depends on the covering of all localities. Finally, simple random sampling was applied to choose the camels from each village. All camels included in this study were aged $<1$ year to $>5$ years. Camels sampled were of both sexes and from local breeds including Bushari, Anafi, Arabi, and Rashide rearing in different ecological areas including desert, arid, savanna, and rich savanna.

2.3. Collection of Blood Samples. Blood samples were collected from jugular vein using plain vacutainer tubes with a needle holder after cleaning the puncture area with $70 \%$ alcohol. A volume of 5 to $7 \mathrm{ml}$ of blood was collected aseptically from each animal, and tubes were left to stand overnight in a refrigerator for serum separation. Serum was decanted in capped vials and frozen at $-20^{\circ} \mathrm{C}$ until transported to the Central Veterinary Research Laboratory for screening.

2.4. Risk Factors. Risk factors investigated in this study included age, sex, breed, locality, and ecology of the area (which were recorded with each serum sample) and subsequently analyzed using Statistical Package for the Social
Sciences (SPSS). Based on ecological features, the study area was divided into four categories, namely, desert, arid, savanna, and rich savanna.

2.5. Laboratory Analysisfor Samples. Indirect enzyme-linked immunosorbent assay (iELISA) was performed using commercially available BTV kit for the detection of specific IgG antibody (Ingizme, France) according to the manufacturer's instructions. In brief, ELISA was performed in 98well antigen-coated microplate. The incubation was performed for $30 \mathrm{~min}$ at room temperature, the plates were then washed six times, conjugate was added and incubated for $45 \mathrm{~min}$, and then the substrate was added and incubated for $10 \mathrm{~min}$. The reaction was stopped using stop solution, and then results were read using ELISA reader (Biochrome, England) set at $630 \mathrm{~nm}$.

2.6. Statistical Analysis. Statistical Package for the Social Sciences (SPSS) software (version 16.0) was used for the analysis of the results. Association between the outcome variables and its risk factor was firstly screened in univariate analysis. Then, multivariable model for the outcome variable was constructed, and BTV infection was considered as dependent variable and the risk factors as independent variables. Finally, odd ratios $95 \%$ at confidence interval were calculated. $p \leq 0.05 \mathrm{p}$ indicated significant association.

\section{Results}

The overall BTV seroprevalence rate in camels was $96.7 \%$, ranging from $93.5 \%$ to $100 \%$ in the six screened localities. No significant association $(p>0.05)$ between BTV seropositivity and age, sex, breed, and locality was found. In the surveyed camel, females and males had similar seroprevalence with estimated $96.7 \%$, unlike age groups which had different BTV seroprevalence rates ranging from $92 \%$ to $100 \%$. In the current study, the most infected camel breeds were Anafi (97.8\%) and Arabi (97.6\%) compared with Bushari (93.0\%) and Rashide (94.4\%) (Table 1). BTV seropositivity assessment according to the ecological area showed high BTV antibody prevalence in desert and savanna with estimated $100 \%$ and lower BTV prevalence in arid and rich savanna with estimated $94.8 \%$ and $95.7 \%$ seroprevalence, respectively (Table 1).

\section{Discussion}

In the present study, no clinical signs were observed in camels, although the overall BTV seroprevalence rate in camels in Gadarif was $96.7 \%$ (ranging from $93.5 \%$ to $100 \%$ in different localities) which was higher than that reported (78.6\%) by Elmahi [13] in the neighboring Kassala state and (66.8\%) by Saeed and Aradaib [10] in Khartoum state. However, earlier seroprevalence surveys showed much lower prevalence in Kassala State (12.7\%) by Hassanin [11] and 6\% by Abu [9] in Gadarif state. Both of these authors used the much less sensitive agar gel immuno-diffusion test (AGID). On the other hand, Chandel et al. [14] in India found the 


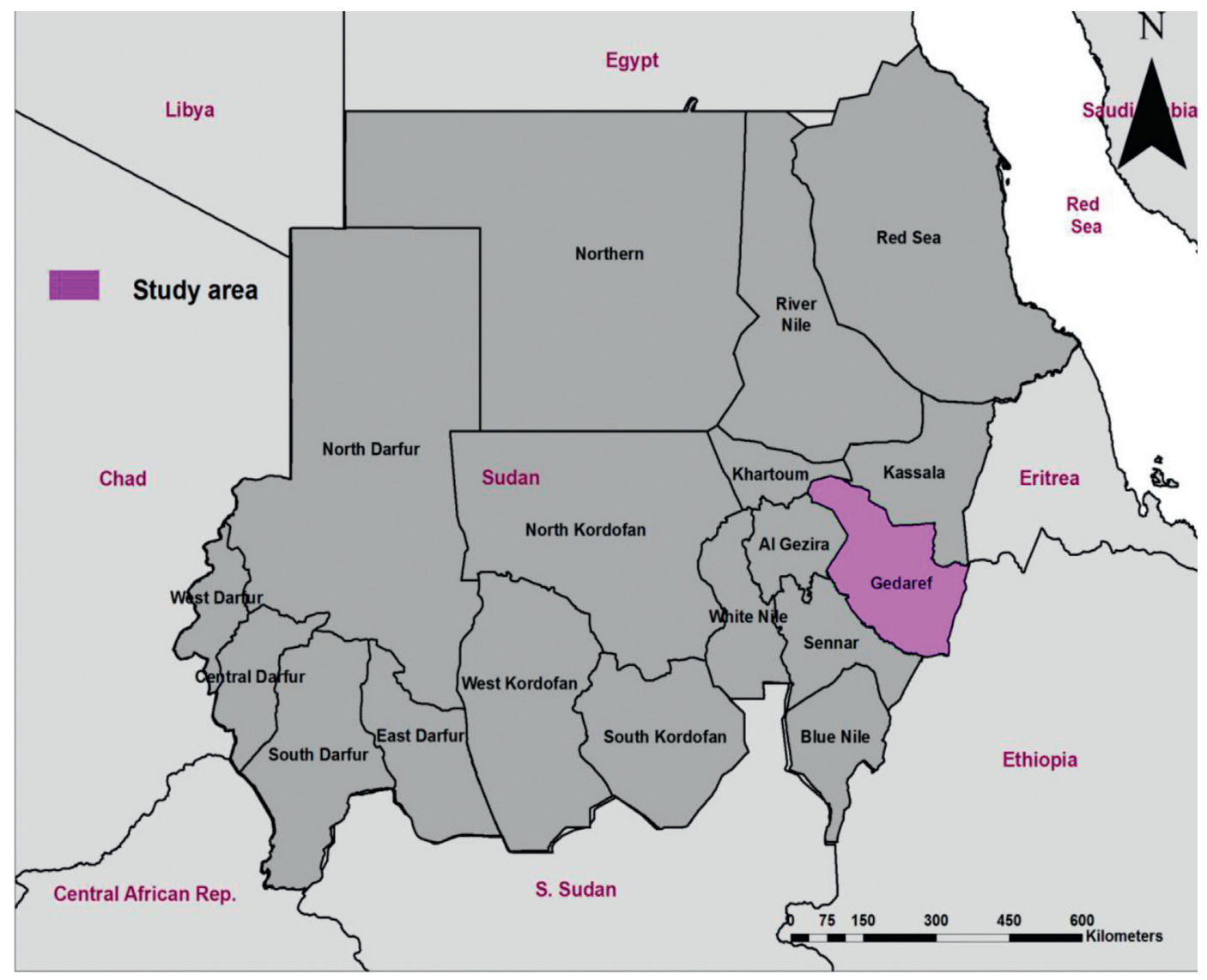

FIGURE 1: Map of Sudan showing the study area (Gadarif state) where serum samples were collected.

TABLE 1: Multivariate analysis for the association between potential risk factors and BTV seropositivity among camels in Gadarif state, eastern Sudan, using chi-square test.

\begin{tabular}{|c|c|c|c|c|c|}
\hline Factor & Animals tested & Animals affected (\%) & $p$ value & Odds ratio & $\begin{array}{c}95 \% \text { CI } \\
\text { Lower-upper }\end{array}$ \\
\hline \multicolumn{6}{|l|}{ Locality } \\
\hline Gadarif & 46 & $43(93.5)$ & Ref & - & - \\
\hline Central Gadarif & 19 & $18(94.7)$ & 0.848 & 1.256 & $0.122-12.896$ \\
\hline Gor Aisha & 46 & $46(100)$ & 0.998 & - & - \\
\hline Fashaga & 32 & $31(96.9)$ & 0.513 & 2.163 & $0.215-21.785$ \\
\hline Butana & 41 & $41(100)$ & 0.998 & - & - \\
\hline Al-Rahad & 92 & $88(95.7)$ & 0.586 & 1.535 & $0.329-7.165$ \\
\hline \multicolumn{6}{|l|}{ Sex } \\
\hline Female & 213 & $206(96.7)$ & Ref & - & - \\
\hline Male & 63 & $61(96.8)$ & 0.965 & 1.036 & $0.210-5.119$ \\
\hline \multicolumn{6}{|l|}{ Age (years) } \\
\hline $0-1$ & 19 & $19(100)$ & 0.998 & - & - \\
\hline $1-2$ & 33 & $32(97.0)$ & 0.406 & 2.667 & $0.264-26.938$ \\
\hline $2-3$ & 17 & $17(100)$ & 0.998 & - & - \\
\hline $3-4$ & 21 & $20(95.2)$ & 0.667 & 1.667 & $0.162-17.100$ \\
\hline $4-5$ & 39 & $36(92.3)$ & Ref & - & - \\
\hline$>5$ & 147 & $143(97.3)$ & 0.165 & 2.979 & 0.638-13.909 \\
\hline \multicolumn{6}{|l|}{ Breed } \\
\hline Bushari & 43 & $40(93.0)$ & Ref & - & - \\
\hline Arabi & 124 & $121(97.6)$ & 0.186 & 3.025 & $0.587-15.590$ \\
\hline Anafi & 91 & $89(97.8)$ & 0.196 & 3.337 & $0.537-20.757$ \\
\hline Rashide & 18 & $17(94.4)$ & 0.838 & 1.275 & $0.124-13.147$ \\
\hline \multicolumn{6}{|l|}{ Ecology } \\
\hline Desert & 41 & $41(100)$ & 0.998 & - & - \\
\hline Arid & 97 & $92(94.8)$ & Ref & - & - \\
\hline Savanna & 46 & $46(100)$ & 0.998 & - & - \\
\hline Rich savanna & 92 & $88(95.7)$ & 0.795 & 1.196 & $0.311-4.598$ \\
\hline
\end{tabular}


estimated seroprevalence rate to be $6.9 \%$ in healthy camels and $12.6 \%$ in sick camels. These findings could be tentative evidence of existence of clinical signs of BTV infection in camels, but more work is needed in Sudan to prove it. Our risk assessment studies indicated that there was no significant association between BTV seropositivity in camels and sex, age, breed, locality, and ecology of the area.

The overall seroprevalence herein reported was higher compared with the seroprevalence rate in Saudi Arabia, Algeria, and Iran which was $25.7 \%, 21 \%$, and $67.8 \%$, respectively [15-17]. This could be due to the degree of exposure of camels to the vectors as well as management practice of keeping them for a long time. The lower seroprevalence of BTV (66.6\%) in camels in Khartoum detected by Saeed and Aradaib [10] compared to our study could be due to the rapid intake of camel in Khartoum state by slaughtering or export as well as low level of vector and/or BTV activity.

There was no significant association $(p>0.05)$ between BTV infection rate noted in this study and ecology of the area. This is in concordance with the findings reported by Elmahi [13] in camels in Kassala state, eastern Sudan. However, the high seroprevalence of BTV in camels in the desert and arid areas in our study (100\%) is unexpectedly very high as these areas may not be suitable to support large populations of Culicoides midges. This could be explained by existence of very localized foci, such as around seasonal water bonds, of Culicoides breeding and survival, or due to movement in and out of the areas. Otherwise, other ways of acquiring the infection such as contact and transplacental transmission as occuring in cattle and goats $[18,19]$ may be working for camels in these areas especially around seasonal water bonds where large numbers of animals closely congregate. Such variables should deserve further investigation.

Our current study revealed no significant association $(p>0.05)$ between the BTV seroprevalence rate and age of animals. This is in line with the finding reported by Elmahi [13] in camels in Kassala state.

\section{Conclusions}

It could be concluded that BTV antibodies are highly prevalent in camels in Gadarif state. No significant association was detected between BTV seropositivity and the risk factors predisposing the animals to the disease. It is recommended that entomological surveillance of biting Culicoides midges involved in the transmission of BTV, and studies of their ecology and epidemiology in the area should also be carried out to better forecast and respond to BT disease in Gadarif state, Sudan.

\section{Data Availability}

The data used to support the findings of this study are available from the corresponding author upon request.

\section{Conflicts of Interest}

The authors declare that there are no conflicts of interest regarding the publication of this paper.

\section{Acknowledgments}

The authors wish to thank the staff of Pathology Department, Central Veterinary Research Laboratory (CVRL), specially Mrs. Zamzam and Mr. Atim, for their great help in serology work. This work was supported by Gadarif Regional Veterinary Laboratory (GRVL), CVRL, and Animal Resources Research Corporation (ARRC).

\section{References}

[1] Z. Farah and A. Fisher, "Milk and meat from the camel handbook on products and processing," 2004.

[2] FAO, Food and Agriculture Organization, FAO, Rome, Italy, 2005.

[3] B. Faye, O. M. A. Abdelhadi, A. I. Ahmed, and S. A. Bakheit, "Camel in Sudan: future prospects," Livestock Research for Rural Development, vol. 23, 10 pages, 2011.

[4] M. E. Abusin, "A change of strategy of animal husbandry among the nomads of Buttana-eastern Sudan," Problem of Agricultural Development in the Sudan, vol. 1, pp. 87-104, 1982.

[5] B. Abbas, G. S. Martin, and D. Planchenauct, "Constrains to camel production in eastern Sudan, a survey of pastoralists conception," Sudan Journal of Veterinary Science and Animal Husbandry, vol. 32, no. 1, pp. 31-41, 1993.

[6] N. J. Maclachlan, C. P. Drew, K. E. Darpel, and G. Worwa, "The pathology and pathogenesis of bluetongue," Journal of Comparative Pathology, vol. 141, no. 1, pp. 1-16, 2009.

[7] W. P. Taylor, "The epidemiology of bluetongue," Revue Scientifique et Technique de l'OIE, vol. 5, no. 2, pp. 351-356, 1986.

[8] D. Verwoerd and B. Erasmus, Bluetongue. Infectious Diseases of Livestock, pp. 1201-1220, Oxford University Press, Oxford, UK, 2nd edition, 2004.

[9] E. M. E. Abu, "Elzein, bluetongue in camels: a serological survey in one humped camel (Camelus dromedaries) in Sudan," Revue d'élevage et de médecine vétérinaire des pays tropicaux, vol. 38, no. 4, pp. 438-442, 1985.

[10] S. I. Saeed and I. E. Aradaib, "A survey of Bluetongue virus antibodies and associate risk factors among camels in Khartoum State, Sudan," Journal of Camel Research and Production, vol. 1, no. 1, pp. 40-49, 2017.

[11] A. A. M. Hassanin, "Epidemiological studies on bluetongue virus activity in eastern sudan," MSc. thesis, University of Khartoum, Khartoum, Sudan, 1994.

[12] M. Eisa, A. E. Karrar, and A. H. A. Elrahim, "Incidence of bluetongue virus precipitating antibodies in sera of some domestic animals in the Sudan," Journal of Hygiene, vol. 83, no. 3, pp. 539-545, 1979.

[13] M. M. Elmahi, "Serosurveillance and molecular detection of bluetongue virus in domestic ruminants in Kassala State, Sudan," MSc. thesis, University of Khartoum, Kassala, Sudan, 2018.

[14] B. S. Chandel, H. C. Chauhan, and H. N. Kher, "Comparison of the standard AGID test and competitive ELISA for detecting of BTV antibodies in camels in India," Tropical Animal Health and Production, vol. 35, no. 2, pp. 99-104, 2003.

[15] M. Yousef, A. AlEesa, and M. AlBlowi, "High seroprevalence of bluetongue virus antibodies in Sheep, Goats, Cattle and Camel in different districts of Saudi Arabia," Veterinary World, vol. 5, no. 7, pp. 389-393, 2012. 
[16] H. Madani, J. Casal, A. Alba et al., "Animal diseases caused by Orbiviruses, Algeria," Emerging Infectious Diseases, vol. 17, no. 12, pp. 2325-2327, 2011.

[17] A. A. Mozaffari, E. Sakhaee, M. Khalili, and A. P. Ardakani, "High seroprevalence of bluetongue virus (BTV) antibodies in camel in Yazd province of Iran," Journal of Camel Practice and Research, vol. 20, no. 2, pp. 171-173, 2013.

[18] F. D. Menzies, S. J. McCullough, I. M. McKeown et al., "Evidence for transplacental and contact transmission of bluetongue virus in cattle," Veterinary Record, vol. 163, no. 7, pp. 203-209, 2008.

[19] C. Batten, K. Darpel, M. Henstock, P. Fay, and E. Veronesi, "Evidence for transmission of bluetongue virus serotype 26 through direct Contact,” PLoS One, vol. 9, no. 5, 2014. 